\title{
Post hoc analysis of two clinical trials to compare the immunogenicity and safety of different polio immunization schedules in Chinese infants
}

\author{
Ting Zhao ${ }^{1 \#}$, Zhaojun $\mathrm{Mo}^{2 \#}$, Zhifang Ying ${ }^{3 \#}$, Teng Huang ${ }^{2}$, Yanchun Che ${ }^{1}$, Guoliang Li ${ }^{1}$, Xiaolei Yang ${ }^{1}$, \\ Mingbo Sun ${ }^{1}$, Li Jiang ${ }^{1}$, Li Shi ${ }^{1}$, Hui Ye ${ }^{1}$, Zhimei Zhao ${ }^{1}$, Xiaochang Liu ${ }^{1}$, Jing Li ${ }^{1}$, Yanping Li $^{2}$, \\ Rongcheng $\mathrm{Li}^{2}$, Ruiju Jiang ${ }^{1}$, Jianfeng Wang ${ }^{3}$, Yuting $\mathrm{Fu}^{1}$, Rufei Ma ${ }^{1}$, Hongyuan Shi ${ }^{1}$, Huan Yang ${ }^{4}$, \\ Changgui Li ${ }^{3}$, Jingsi Yang ${ }^{1}$, Qihan $\mathrm{Li}^{1}$
}

${ }^{1}$ Institute of Medical Biology, Chinese Academy of Medical Sciences and Peking Union Medical College, Kunming, China; ${ }^{2}$ Guangxi Province Center for Disease Control and Prevention, Nanning, China; ${ }^{3}$ National Institutes for Food and Drug Control, Beijing, China; ${ }^{4}$ Center for Drug Evaluation, China Food and Drug Administration, Beijing, China

Contributions: (I) Conception and design: Q Li, J Yang, C Li; (II) Administrative support: H Yang; (III) Provision of study materials or patients: Z Mo; (IV) Collection and assembly of data: T Zhao, Z Mo, H Ye, Z Zhao, X Liu, J Li, Y Li, R Li, R Jiang, J Wang, Y Fu, R Ma, H Shi; (V) Data analysis and interpretation: T Zhao, Z Ying, T Huang, Y Che, G Li, X Yang, M Sun, L Jiang, L Shi; (VI) Manuscript writing: All authors; (VII) Final approval of manuscript: All authors.

"These authors contributed equally to this work.

Correspondence to: Qihan Li, PhD. Institute of Medical Biology, Chinese Academy of Medical Sciences and Peking Union Medical College, 935 Jiaoling Road, Kunming 650118, China. Email: liqihan@imbcams.com.cn; Jingsi Yang, MBBS. Institute of Medical Biology, Chinese Academy of Medical Sciences and Peking Union Medical College, 935 Jiaoling Road, Kunming 650118, China. Email: yjs@imbcams.com.cn; Changgui Li, PhD. 31 Huatuo Road, Daxing District, Beijing 100000, China. Email: changguili@aliyun.com; Huan Yang, PhD. 128 Jianguo Road, Chaoyang District, Beijing 100000, China. Email: 13691354049@163.com.

Background: A comparative analysis of the immunogenicity and safety of different poliovirus immunization schedules in Chinese infants is imperative to guide the administration of efficient strategies for the eradication of poliomyelitis.

Methods: A post boc analysis was conducted with the data from two poliovirus vaccine clinical trials involving a combined total of 2,400 infants aged 60-90 days. Trivalent oral poliovirus vaccine (tOPV), bivalent oral poliovirus vaccine (bOPV), Sabin strain-based inactivated poliovirus vaccine (sIPV), and conventional inactivated poliovirus vaccine (cIPV) were used in different schedules, the immunogenicity and safety of which were compared 28 days after the last of three doses.

Results: In a per-protocol set analysis, the tOPV-tOPV-tOPV schedule induced seroconversion in $99.1 \%, 98.2 \%$, and $96.0 \%$ of the inoculated infants for poliovirus type I, II, and III, respectively. The seroconversions for poliovirus types I and III were each almost $100 \%$ after immunization with the cIPVbOPV-bOPV, sIPV-sIPV-bOPV, cIPV-cIPV-bOPV, sIPV-sIPV-tOPV, cIPV-cIPV-tOPV, or sIPV-bOPVbOPV schedule. However, the schedules that used one IPV dose followed by two (poliovirus type I and III) bOPV doses failed to induce high-level immunity against type II poliovirus. IPV-related schedules were associated with a slightly higher incidence of adverse events (AEs).

Conclusions: If the capacity of IPV can be increased, two or more doses of IPV should be administered before vaccination with bOPV in a sequential schedule to improve immunity against type II poliovirus.

Keywords: Poliovirus sequential vaccination schedule; oral poliovirus vaccine (OPV); inactivated poliovirus vaccine (IPV); immunogenicity; safety

Submitted Mar 16, 2020. Accepted for publication Oct 12, 2020.

doi: $10.21037 / \mathrm{atm}-20-2537$

View this article at: http://dx.doi.org/10.21037/atm-20-2537 


\section{Introduction}

The worldwide use of attenuated poliovirus vaccine (Sabin strains) has contributed greatly to the eradication of infections with wild polioviruses in children (1), but the existence of vaccine-derived polioviruses (VDPVs) and vaccine-associated paralytic poliomyelitis (VAPP) remains an obstacle in the final stage of poliomyelitis eradication $(2,3)$. The switch from oral poliovirus vaccine (OPV) to inactivated poliovirus vaccine (IPV) was proposed as an important strategy for realizing the goal of eradicating poliomyelitis (4). However, although the implementation of this strategy has resulted in fewer cases caused by wild poliovirus strains, the existence of VDPVs still negatively impacts poliomyelitis eradication efforts $(5,6)$. In this context, the World Health Organization (WHO) proposed a strategy of implementing a phased withdrawal of trivalent OPV (tOPV) and introducing IPV; this plan includes one or two doses of IPV to induce basic immunity against poliovirus, especially type II poliovirus, followed by one or two doses of bivalent OPV (bOPV) as a boost (2). It is designed to further decrease the number of poliomyelitis cases induced by wild poliovirus strains and reduce the likelihood of infection with VDPVs. This transitional strategy is expected to produce a suitable environment for the complete switch from immunization schedules that incorporate OPV to an IPV-only immunization schedule (7).

The experts and policy makers in China have been concerned about the immune effect of using immunization schedules that combine IPV and OPV during the switch from a tOPV schedule to an IPV-bOPV schedule. Sabin strain-based inactivated poliovirus vaccine (sIPV) was recently licensed in China in 2015, so there are fewer data about the use of sIPV in sequential immunization. Two clinical trials have been performed to investigate the efficacy and safety of sequential immunization with sIPV and bOPV in China. Because altering the poliovirus vaccination approach from using only tOPV to using a sequential schedule that combines IPV and bOPV will result in changes to immunity against poliovirus in infants, we performed a post hoc analysis of two clinical trials to observe these changes. The resulting data will provide guidance for the application of sequential poliovirus immunization in China.

The post boc analysis performed here was designed to compare and evaluate the immunogenicity and safety of different poliovirus immunization schedules based on the results from two clinical trials. These trials both investigated the immune effects of a three-tOPV-dose schedule and of a schedule composed of one or two IPV doses followed by two or one bOPV doses in children aged 2, 3, and 4 months. The seroconversion rates and neutralizing antibody titers in the vaccinated infants were compared to evaluate poliovirus vaccine performance during the switch from a tOPV immunization schedule to an IPV-bOPV immunization schedule in China.

\section{Methods}

\section{Study design}

This post hoc analysis included two clinical trials. One trial was conducted in Guangxi Province, China from 20112012 to evaluate the safety and immunogenicity of the live attenuated OPV (human diploid cell) (ClinicalTrials.gov number: NCT02231632). The other trial was carried out from 2015-2016 in Guangxi Province, China to examine the immunogenicity and safety of sequential immunization schedules of IPV+bOPV (ClinicalTrials.gov number: NCT03614702). Both trials were sponsored by the Institute of Medical Biology, Chinese Academy of Medical Sciences (IMBCAMS), and Guangxi Center for Disease Prevention and Control. Both clinical studies were conducted in accordance with the Declaration of Helsinki (as revised in 2013) and were approved by the Ethical Committee of Guangxi Zhuang Autonomous Region (approval numbers: 2009L07791 and GXIRB2015-0024-01). Informed consent was obtained for all included participants.

\section{Participants}

The inclusion and exclusion criteria in both clinical trials were similar. The inclusion criteria were as follows: (I) infant is younger than 90 days but older than 60 days; (II) guardian provides written informed consent; (III) the infant's guardian and family follow the requirements of the clinical trial protocol; (IV) infant has no immune globulin immunization history after birth (except hepatitis B immune globulin) and no history of other live vaccination in the 28 days before vaccination; and (V) infant has an axillary temperature of $<37.1{ }^{\circ} \mathrm{C}$. The exclusion criteria were as follows: (I) infant has a personal or family history of allergy, convulsions, epilepsy, encephalopathy, or psychosis; (II) infant has an allergy to neomycin, streptomycin, or polymyxin B; (III) infant has an immunodeficiency or is receiving immunosuppressors; (IV) infant has a history of poliomyelitis; (V) infant has an acute febrile disease or infectious disease; (VI) infant experiences an abnormal stage 
of labor, has a history of asphyxiation, or has a congenital malformation, developmental disorder, or severe chronic disease; (VII) infant has exhibited severe anaphylactic reactions following any previous vaccination; (VIII) infant has received oral steroids for 14 consecutive days within 1 month before the trial; (IX) infant has had a fever (axillary temperature of $\geq 38.0^{\circ} \mathrm{C}$ ) in the previous 3 days; $(\mathrm{X})$ infant has had diarrhea (defecation frequency of $\geq 3$ times/day) within the previous week; (XI) infant is participating in other clinical drug trials; and (XII) infant has any other condition that might influence the evaluation. The clinical trial that was conducted to examine the immunogenicity and safety of sequential immunization schedules composed of IPV and bOPV had one more inclusion criterion: infant had no history of immunization with an inactivated vaccine in the 14 days before vaccination.

The guardians and families of the participants voluntarily complied with the requirements of the clinical trial protocol. An informed consent form was signed by both the guardians and the study doctor of each participant prior to initiation of the clinical trial. Participants were permitted to voluntarily withdraw at any time during the trial. Participants could be withdrawn from the study in cases of failure to adhere to the follow-up visits, violation of or deviation from the trial protocol, or the appearance of other abnormal symptoms that interfered with the trial.

\section{Vaccines}

In clinical trial NCT02231632, the administered tOPV was derived from the human embryonic lung diploid cell line KMB-17 or the monkey kidney cell line Vero (provided by IMBCAMS). The total amount of live poliovirus was no less than $6.15 \operatorname{lgCCID}_{50}(50 \%$ cell culture infective dose); each dose of tOPV included type I ( $\left.\geq 6.0 \operatorname{lgCCID}{ }_{50}\right)$, type II $\left(\geq 5.0 \operatorname{lgCCID}{ }_{50}\right)$, and type $\mathrm{III}\left(\geq 5.5 \operatorname{lgCCID}_{50}\right)$ poliovirus.

In clinical trial NCT03614702, the SIPV was provided by IMBCAMS. Each dose $(0.5 \mathrm{~mL})$ contained 30, 32, and $45 \mathrm{D}$-antigen units of poliovirus types I, II, and III, respectively. The conventional inactivated poliovirus vaccine (cIPV) was purchased from Sanofi Pasteur. Each dose $(0.5 \mathrm{~mL})$ contained 40,8 , and $32 \mathrm{D}$-antigen units of poliovirus types I, II, and III, respectively. The bOPV was provided by IMBCAMS. Each bOPV dose contained at least $5.92 \operatorname{lgCCID}_{50}$ of live poliovirus, comprising type I $\left(\geq 5.8 \operatorname{lgCCID}{ }_{50}\right)$ and type III $\left(\geq 5.3 \operatorname{lgCCID}_{50}\right)$ polioviruses. The $\mathrm{tOPV}$ was derived from the human embryonic lung diploid cell line KMB-17 as in clinical trial NCT02231632.

\section{Procedures}

In clinical trial NCT02231632, the eligible infants $(\mathrm{N}=1,200)$ randomly received three doses of either the tOPV from human diploid cells or the tOPV from monkey kidney cells at the age of 2, 3, and 4 months in accordance with the routine poliovirus vaccine inoculation procedure in China.

In clinical trial NCT03614702, 1,200 participants were randomized to receive one of six different sequential poliovirus immunization schedules (sIPV-bOPV-bOPV, sIPV-sIPV-bOPV, sIPV-sIPV-tOPV, cIPV-bOPV-bOPV, cIPV-cIPV-bOPV, or cIPV-cIPV-tOPV; 200 participants per group). The IPV was administered via an intramuscular injection, whereas the OPV was administered orally. The three vaccine doses were administered at ages 2,3 , and 4 months old, respectively.

In accordance with the approved protocol, participants were observed onsite for $30 \mathrm{~min}$ after vaccination. In clinical trial NCT02231632, any adverse events (AEs) occurring up to 30 days after vaccination were recorded. In clinical trial NCT03614702, AEs were recorded through 28 days post-inoculation, and any serious AEs (SAEs) that occurred between 28 days and 6 months after full vaccination were followed up. Venous blood samples were collected before the first dose and at 28 days after the third dose and used by the National Institutes for Food and Drug Control to test the titer and seroconversion rate of antibodies following the protocol recommended by the WHO. Seroconversion was defined as a neutralizing antibody titer of $<1: 8$ before vaccination and of $>1: 8$ against poliovirus types I, II, and III poliovirus after three doses of vaccine. A neutralizing antibody titer of $\geq 1: 8$ before vaccination was considered to indicate maternally-transferred antibodies; for these cases, seroconversion was defined as a four-fold increase of poliospecific antibody response after three doses of vaccine. The changes in antibody titer after vaccination were analyzed. Both the maximum dilution and the maximum reported titer were 16,384; in cases where the actual titer was greater than 16,384 , the value used in calculations was 16,384 .

\section{Statistical analysis}

The seroconversion rates against poliovirus types I, II, and III were calculated in terms of the poliovirus immunization schedule. The seroconversion rates and titers of antibodies against poliovirus types I, II, and III were compared between the groups of infants vaccinated under different 


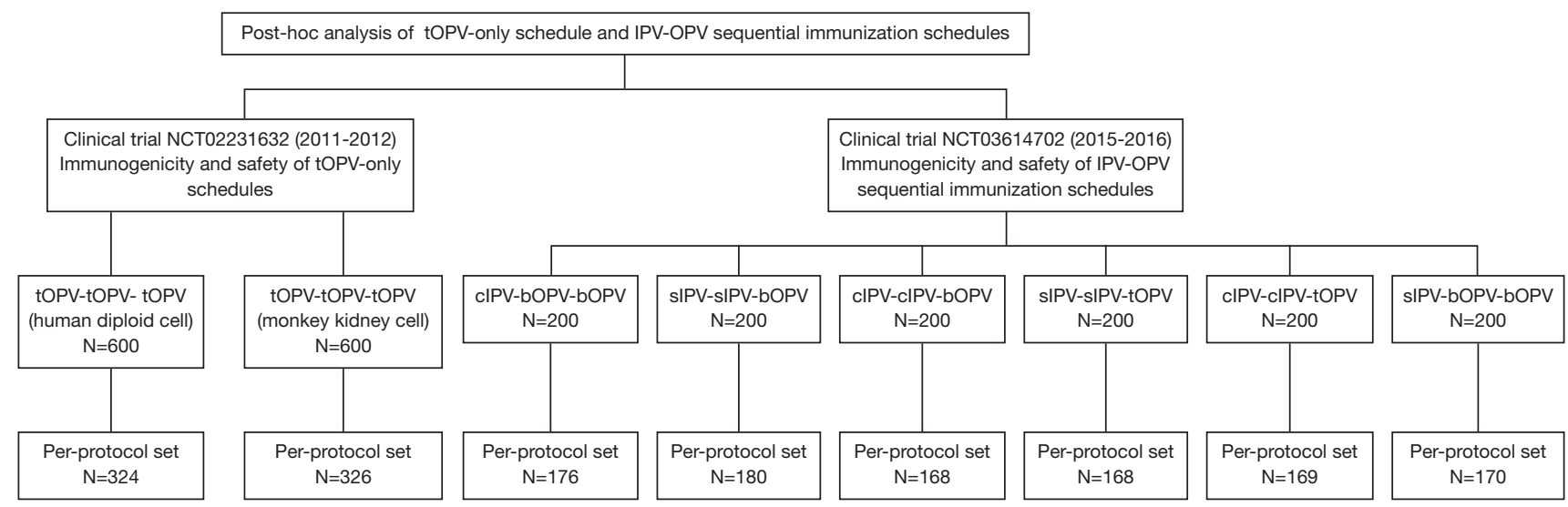

Figure 1 Data flow chart of the two clinical trials included in this post hoc analysis. bOPV, bivalent (types I and III) oral polio vaccine; IPV, inactivated polio vaccine; tOPV, trivalent oral polio vaccine; sIPV; Sabin strain-based IPV; cIPV, conventional IPV, also known as Salk strainbased IPV.

schedules, and the significance of each difference was tested with a two-sided $95 \%$ confidence interval. A chi-squared test was used to compare the differences in the titer of poliovirus type II-specific antibodies between groups.

The post-vaccination geometric mean titer (GMT) and the fold changes of antibodies against poliovirus types I, II, and III in all groups are presented as the geometric means and $95 \%$ confidence intervals. The differences between groups were compared by performing an analysis of variance after logarithmic transformation of the data.

\section{Results}

\section{Baseline characteristics of the vaccinated infants}

This post hoc analysis was based on the data from two clinical trials conducted in Guangxi Province, China (Figure 1). The baseline characteristics of the infants in the per protocol set (PPS) analysis were similar between groups (Table 1, Table S1).

\section{Seroconversion rates induced by poliovirus immunization schedules}

The seroconversion rates against poliovirus types I, II, and III were $99.1 \%, 98.2 \%$, and $96.0 \%$, respectively, in the infants who received three tOPV doses (Table 2). Notably, the positive rates of antibodies against poliovirus types I, II, and III before vaccination were 49.2\%, 30.3\%, and $24.0 \%$, respectively; these positive cases are likely due to maternal antibodies (Table 1) (8). For the combined sequential immunization programs, the IPV-bOPV-bOPV immunization program had a lower seroconversion rate against poliovirus type II than did the IPV-IPV-bOPV immunization program, regardless of whether the IPV used in the immunization program was sIPV or cIPV (Table 2, Tables S2,S3). However, the seroconversion rates for poliovirus type I and III were both almost $100 \%$ for all sequential schedule groups. The modestly higher seroconversion rates for poliovirus type III in the IPVbOPV-bOPV groups and IPV-IPV-bOPV groups suggest that bOPV can induce a better immune response to poliovirus type III compared with tOPV because it lacks the competition from poliovirus type II. The seroconversion rates against poliovirus type II were $51.8 \%$ in the sIPVbOPV-bOPV group and $60.2 \%$ in the cIPV-bOPV-bOPV group, suggesting that these schedules confer insufficient clinical protection against wild poliovirus or VDPV strains (Table 2). However, compared with the schedules containing one IPV dose, the vaccination schedules containing two IPV doses (sIPV or cIPV) followed by one bOPV dose were capable of eliciting a higher seroconversion rate to poliovirus type II (79.4\% of vaccinated infants for sIPVsIPV-bOPV and $81.0 \%$ for cIPV-cIPV-bOPV), suggesting that a single antigenic stimulation by the poliovirus type II vaccine is unable to elicit adequate immune protection. Thus, two or more IPV doses should be required, especially for infants at a high risk of infection with type II VDPV.

\section{Neutralizing antibody titers}

The effectiveness of the immunologic barrier against 


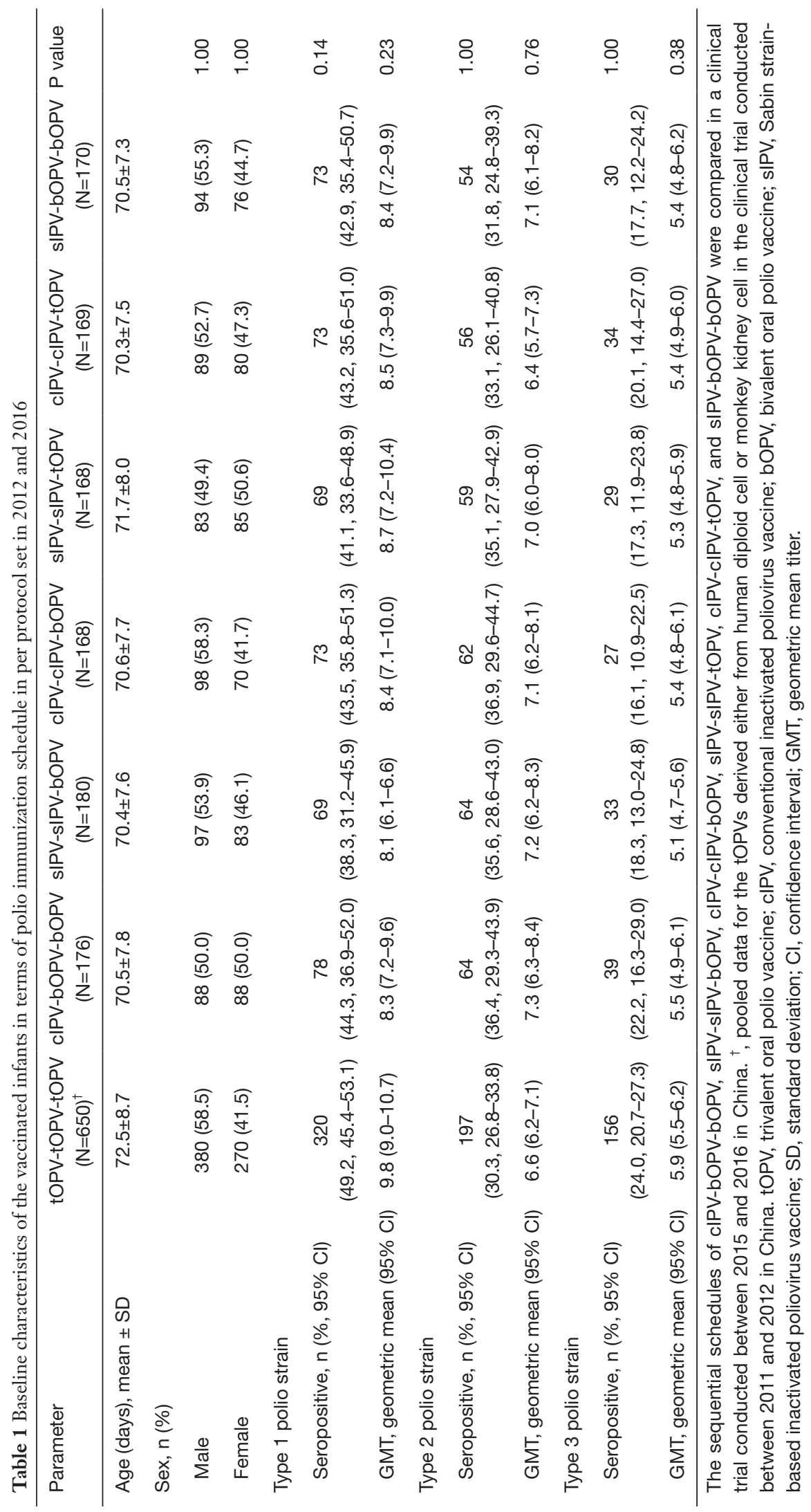




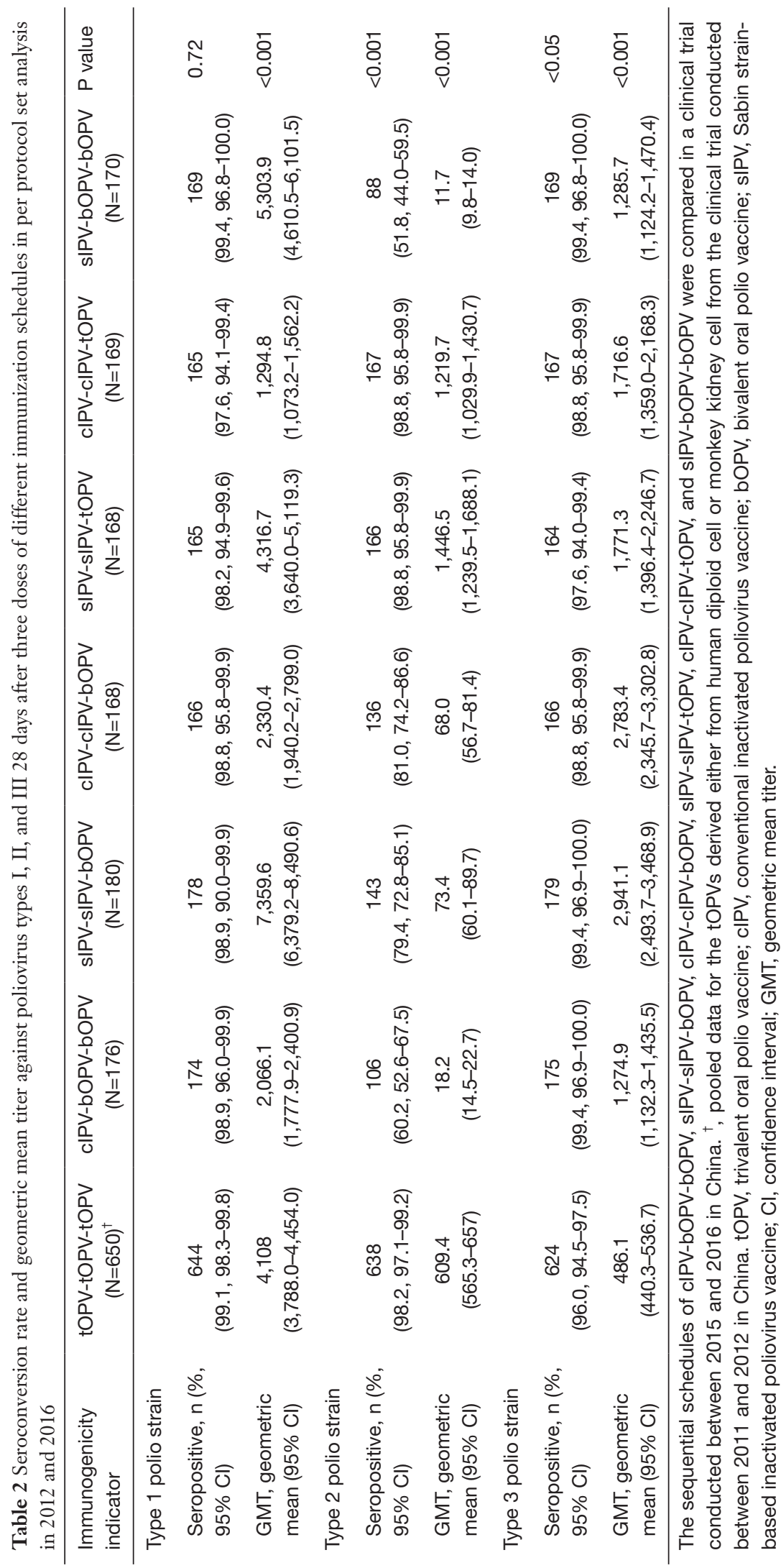


Type 1

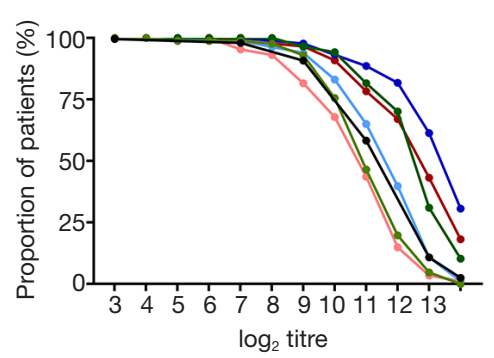

Type 2

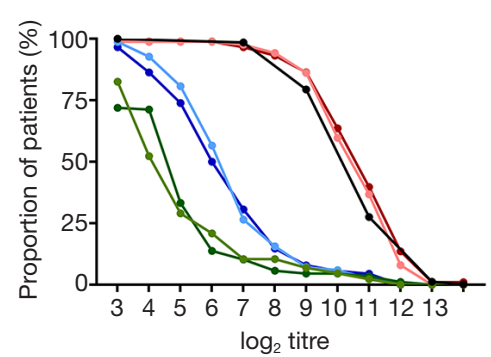

Type 3

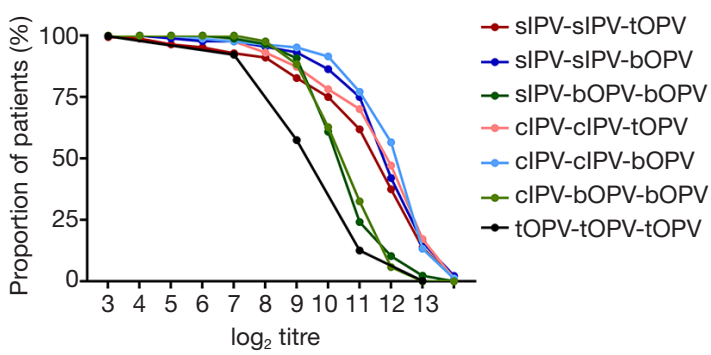

Figure 2 Reverse cumulative distribution curves showing the titers of serum antibodies neutralizing poliovirus types I, II and III.

infection by wild poliovirus or VDPV strains largely depends on the level of neutralizing antibodies in the sera of an individual $(9,10)$. We observed high GMTs for all three types of poliovirus after immunization with tOPVtOPV-tOPV, which is consistent with a previous report (11). The IPV-bOPV-bOPV and IPV-IPV-bOPV schedules, regardless of whether sIPV or cIPV was used, were capable of eliciting high GMTs against poliovirus types I and III (Table 2, Figure 2). However, the IPV-bOPV-bOPV schedule induced a lower level of antibody response against type II poliovirus, regardless of whether sIPV or cIPV was used. Although the IPV-IPV-bOPV group exhibited a relatively high level of antibodies against type II poliovirus, this level was still lower than those in the IPV-IPV-tOPV groups (Table 2, Table S4, Figure 2).

\section{Safety evaluation}

The safety of the tOPV-tOPV-tOPV schedule has been demonstrated over its $>50$ years of use in Chinese children (12); the primary safety concern for this approach is the rare cases of VAPP $(6,13)$. As part of new poliovirus eradication strategy, the final goal of which is to adopt an IPV-only immunization schedule and eradicate all wild polioviruses and VDPVs, a switch from tOPV to bOPV is necessary, and the safety of this change must be evaluated. One of the included clinical trials reported a total of 951 AEs, and the other reported 918 AEs (Table 3). The incidences of fever were $28.0 \%(335 / 1,195)$ in the tOPV-tOPV-tOPV schedule and 49.9\% $(598 / 1,198)$ for all sequential schedules combined. Additionally, the incidences of abnormal irritability were $5.9 \%$ and $20.9 \%$, the incidences of local reactions (e.g., pain, redness, swelling, and induration) were $0 \%$ and $1.3 \%$, and the incidences of gastrointestinal symptoms were $10.0 \%$ and $6.7 \%$ in the
tOPV-tOPV-tOPV schedule and for all sequential schedules combined, respectively. SAEs were reported in 5.9\% $(71 / 1,195)$ of participants in the tOPV-tOPV-tOPV group and in $5.0 \%(60 / 1,198)$ of participants who were vaccinated with a sequential schedule (Table 3, Table S5). The injected IPV was associated with a relatively high incidence of AEs, even though most of the reported AEs were identified as being unrelated to inoculation with poliovirus vaccine.

\section{Discussion}

The sequential removal of Sabin poliovirus strains leading up to a full replacement of OPV with IPV is a goal established by the Global Polio Eradication Initiative [2015] for the eradication of poliomyelitis caused by all wild polioviruses and VDPVs in children worldwide. As the first step towards this goal, the strategy of using sequential immunization schedules combining bOPV with IPV by removing Sabin type II should maintain an adequate immune protective effect against any possible infection by wild poliovirus strains or VDPVs (14).

From 2011 to 2012, we conducted a clinical trial to evaluate the safety and immunogenicity of the live attenuated OPV (human diploid cell; ClinicalTrials. gov number: NCT02231632). Vaccination with three doses of tOPV administered at 2, 3, and 4 months of age, respectively, was the immunization strategy against poliomyelitis in mainland China until April 2016 (15). Since then, global immunization strategies for poliovirus have changed. Clinical trial NCT03614702 was carried out from 2015 to 2016 in Guangxi Province, China to examine the immunogenicity and safety of sequential immunization schedules with sIPV+bOPV. The present study is a comprehensive analysis of these two clinical trials in which the changes induced by switching the immunization strategy 


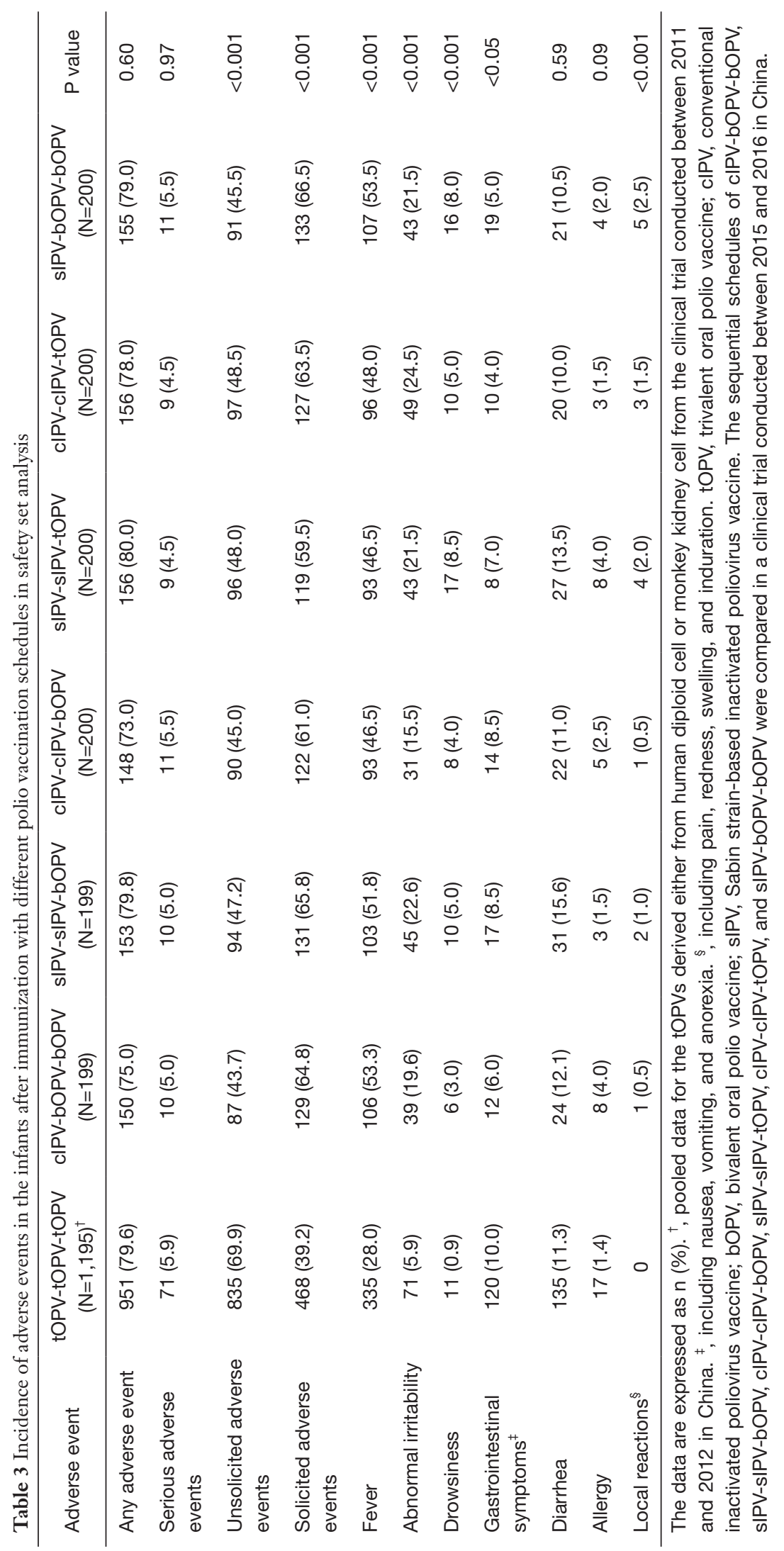


from only tOPV to IPV+bOPV can be observed. Although some published reports describe other clinical trials on sequential immunization with IPV and OPV, most of the previous studies on sequential poliovirus immunization used conventional IPV and did not incorporate the use of sIPV. Our study supplements the existing data by addressing the use of sIPV in sequential immunization.

This post hoc analysis compared the immune effects induced by a tOPV-tOPV-tOPV schedule with those induced by various sequential schedules using IPV (sIPV or cIPV) followed by bOPV. Vaccine-induced immunity to poliovirus must be maintained at an adequate level in Chinese children during the transition from IPV-bOPV combination schedules to the complete cessation of OPV administration and the sustained use of an IPV-only schedule. Our results confirm that, similar to the tOPVtOPV-tOPV schedule, schedules containing one or two IPV doses (sIPV or cIPV) followed by one or two doses of bOPV could induce high seroconversion rates against poliovirus type I and III, associated with high neutralizing antibody titers. However, the administration of one IPV dose followed by two doses of bOPV failed to induce an adequate level of seroconversion or neutralizing antibody titer against type II poliovirus. Thus, the application of a schedule with a single IPV dose during the transition period may lead to an increased risk of infection with type II circulating vaccine-derived poliovirus (cVDPV). In contrast, the administration of two IPV doses followed by one dose of bOPV elicit higher levels of seroconversion and antibody titers against type II poliovirus.

There are still pandemics of infection with wild poliovirus strains in at least two countries that border China $(5,16)$. To improve immunity against type II poliovirus, two or more doses of IPV should be administered before the administration of bOPV in a sequential schedule. A previous study of IPV also reported that one dose of IPV induced seroconversion rates of $19-46 \%, 32-63 \%$, and $28-54 \%$ for poliovirus types I, II, and III, respectively (17). A boost with poliovirus vaccine is important for the establishment of effective immunity against poliovirus $(18,19)$. Importantly, all these data suggest that a schedule using one dose of IPV followed by two doses of bOPV is not suitable for poliovirus immunization during the transition period, especially in developing countries where tOPV had been used alone for a long time and where there is still a risk of the spread of wild poliovirus strains and VDPVs $(20,21)$. At this stage, using at least two doses of IPV will be a better option for a routine poliovirus immunization schedule to maintain an adequate level of population immunity to all poliovirus types and avoid the emergence of epidemic cases of paralysis induced by wild poliovirus strains or VDPVs.

The safety evaluation results suggest that sequential immunization schedules combining OPV with IPV are associated with a higher incidence of some AEs, such as fever and local reactions, which may be due to the inclusion of one or two injections in these schedules. Although both included clinical trials were completed in the same region, they were not conducted during the same period, and there were also some small differences in their methods of $\mathrm{AE}$ collection, so the $\mathrm{AE}$ differences between poliovirus vaccination schedules may be caused by factors other than the immunization procedure.

In conclusion, during the transition from a tOPV-only immunization schedule to an IPV-bOPV immunization schedule, sequential schedules containing two IPV doses are a reasonable option that align with current WHO recommendations. Our findings may help decision-makers in developing countries to better optimize poliovirus vaccination policies.

\section{Acknowledgments}

We are very grateful to the staff of the Centers for Disease Control and Prevention of Guangxi Province and Liujiang City and all the onsite sub-investigators for their contributions. We are also indebted to the staff of National Institutes for Food and Drug Control for providing the serum neutralization antibody assay, to Simurike Pharmaceutical Information Consulting Co., Ltd. for supervising the study and providing data management services, and to the experts from the Center for Drug Evaluation, China Food and Drug Administration and National Center for Disease Control and Prevention for their valuable opinions and suggestions. We thank Katie Oakley, PhD, from Liwen Bianji, Edanz Editing China (www.liwenbianji.cn/ac), for editing the English text of a draft of this manuscript.

Funding: This work was supported by the State Project for Essential Drug Research and Development (2015ZX09101031) and Yunnan Province Project (2016BC002).

\section{Footnote}

Data Sharing Statement: Available at http://dx.doi. org/10.21037/atm-20-2537 
Peer Review File: Available at http://dx.doi.org/10.21037/ atm-20-2537

Conflicts of Interest: All authors have completed the ICMJE uniform disclosure form (available at http://dx.doi. org/10.21037/atm-20-2537). The authors have no conflicts of interest to declare.

Ethical Statement: The authors are accountable for all aspects of the work in ensuring that questions related to the accuracy or integrity of any part of the work are appropriately investigated and resolved. The two included clinical studies were each conducted in accordance with the Declaration of Helsinki (as revised in 2013). These two clinical studies were approved by the Ethical Committee of Guangxi Zhuang Autonomous Region (approval numbers: 2009L07791 and GXIRB2015-0024-01), and informed consent was obtained for all the participants.

Open Access Statement: This is an Open Access article distributed in accordance with the Creative Commons Attribution-NonCommercial-NoDerivs 4.0 International License (CC BY-NC-ND 4.0), which permits the noncommercial replication and distribution of the article with the strict proviso that no changes or edits are made and the original work is properly cited (including links to both the formal publication through the relevant DOI and the license). See: https://creativecommons.org/licenses/by-nc-nd/4.0/.

\section{References}

1. Thompson KM, Tebbens RJ. Current polio global eradication and control policy options: perspectives from modeling and prerequisites for oral poliovirus vaccine cessation. Expert Rev Vaccines 2012;11:449-59.

2. Patel M, Zipursky S, Orenstein W, et al. Polio endgame: the global introduction of inactivated polio vaccine. Expert Rev Vaccines 2015;14:749-62.

3. Duintjer Tebbens RJ, Pallansch MA, Kim JH, et al. Oral poliovirus vaccine evolution and insights relevant to modeling the risks of circulating vaccine-derived polioviruses (cVDPVs). Risk Anal 2013;33:680-702.

4. Garon J, Seib K, Orenstein WA, et al. Polio endgame: the global switch from tOPV to bOPV. Expert Rev Vaccines 2016;15:693-708.

5. Initiative GPE. List of wild poliovirus by country and year. 2019. Available online: http://www.polioeradication.org/ Dataandmonitoring/Poliothisweek/Wildpolioviruslist. aspx. Accessed 1 Jul 2019.

6. Wang HB, Luo HM, Li L, et al. Vaccine-derived poliovirus surveillance in China during 2001-2013: the potential challenge for maintaining polio free status. BMC Infect Dis 2017;17:742.

7. Initiative GPE. Polio Eradication \& Endgame Strategic Plan 2013-2018. 2016. Available online: http://www. polioeradication.org/Portals/0/Document/Resources/ StrategyWork/PEESP_EN_US.pdf. Accessed 12 Jul 2019.

8. Gaensbauer JT, Gast C, Bandyopadhyay AS, et al. Impact of Maternal Antibody on the Immunogenicity of Inactivated Polio Vaccine in Infants Immunized With Bivalent Oral Polio Vaccine: Implications for the Polio Eradication Endgame. Clin Infect Dis 2018;67:S57-S65.

9. Bandyopadhyay AS, Garon J, Seib K, et al. Polio vaccination: past, present and future. Future Microbiol 2015;10:791-808.

10. Norrby E, Uhnoo I, Brytting $M$, et al. Polio close to eradication. Lakartidningen 2017;114:EPDT.

11. Lu L, Li X, Zhang H, et al. Immunogenicity and persistence from different 3 -dose schedules of live and inactivated polio vaccines in Chinese infants. Vaccine 2015;33:4653-8.

12. Yu WZ, Wen N, Zhang Y, et al. Poliomyelitis eradication in China: 1953-2012. J Infect Dis 2014;210 Suppl 1:S268-74.

13. Yan D, Zhang Y, Zhu S, et al. Limited and localized outbreak of newly emergent type 2 vaccine-derived poliovirus in Sichuan, China. Clin Vaccine Immunol 2014;21:1012-8.

14. Platt LR, Estivariz CF, Sutter RW. Vaccine-associated paralytic poliomyelitis: a review of the epidemiology and estimation of the global burden. J Infect Dis 2014;210 Suppl 1:S380-9.

15. Prevention CCfDCa. Decision to Implement New Polio Vaccine Immunization Strategy. 2016. Available online: http://www.chinacdc.cn/jkzt/crb/zl/jshzy/. Accessed 5 Jul 2019.

16. Yan D, Wang D, Zhu S, et al. Immunogenicity of oral polio vaccine and Salk inactive polio vaccine against Xinjiang imported type 1 wild poliovirus. Clin Infect Dis 2020;70:1980-4.

17. Estivariz CF, Pallansch MA, Anand A, et al. Poliovirus vaccination options for achieving eradication and securing the endgame. Curr Opin Virol 2013;3:309-15.

18. O'Ryan M, Bandyopadhyay AS, Villena R, et al. Inactivated poliovirus vaccine given alone or in a sequential schedule with bivalent oral poliovirus vaccine in Chilean infants: a randomised, controlled, open-label, phase 4, non- 
inferiority study. Lancet Infect Dis 2015;15:1273-82.

19. Taniuchi M, Famulare M, Zaman K, et al. Community transmission of type 2 poliovirus after cessation of trivalent oral polio vaccine in Bangladesh: an open-label clusterrandomised trial and modelling study. Lancet Infect Dis 2017;17:1069-79.

20. Lewis I, Ottosen A, Rubin J, et al. A Supply and Demand

Cite this article as: Zhao T, Mo Z, Ying Z, Huang T, Che Y, Li G, Yang X, Sun M, Jiang L, Shi L, Ye H, Zhao Z, Liu X, Li J, Li Y, Li R, Jiang R, Wang J, Fu Y, Ma R, Shi H, Yang H, Li C, Yang J, Li Q. Post hoc analysis of two clinical trials to compare the immunogenicity and safety of different polio immunization schedules in Chinese infants. Ann Transl Med 2021;9(3):253. doi: 10.21037/atm-20-2537
Management Perspective on the Accelerated Global Introductions of Inactivated Poliovirus Vaccine in a Constrained Supply Market. J Infect Dis 2017;216:S33-9.

21. Duintjer Tebbens RJ, Hampton LM, Thompson KM. Implementation of coordinated global serotype 2 oral poliovirus vaccine cessation: risks of potential nonsynchronous cessation. BMC Infect Dis 2016;16:231. 


\section{Supplementary}

Table S1 Statistical analysis of baseline characteristics of the vaccinated infants in terms of polio immunization schedule in per protocol set

\begin{tabular}{|c|c|c|}
\hline Parameter & Test method & $P$ value \\
\hline \multicolumn{3}{|l|}{ Age (days), mean \pm SD } \\
\hline \multicolumn{3}{|l|}{ Sex, n (\%) } \\
\hline Male & Pearson CHI-SQUARE & 1.00 \\
\hline Female & Pearson CHI-SQUARE & 1.00 \\
\hline \multicolumn{3}{|l|}{ Type 1 polio strain } \\
\hline Seropositive, n (\%, 95\% Cl) & Pearson CHI-SQUARE & 0.14 \\
\hline GMT, geometric mean $(95 \% \mathrm{Cl})$ & ANOVA & 0.23 \\
\hline \multicolumn{3}{|l|}{ Type 2 polio strain } \\
\hline Seropositive, n (\%, 95\% Cl) & Pearson CHI-SQUARE & 1.00 \\
\hline GMT, geometric mean $(95 \% \mathrm{Cl})$ & ANOVA & 0.76 \\
\hline \multicolumn{3}{|l|}{ Type 3 polio strain } \\
\hline Seropositive, n (\%, 95\% Cl) & Pearson CHI-SQUARE & 1.00 \\
\hline GMT, geometric mean $(95 \% \mathrm{Cl})$ & ANOVA & 0.38 \\
\hline
\end{tabular}

$\mathrm{SD}$, standard deviation; $\mathrm{Cl}$, confidence interval; GMT, geometric mean titer; ANOVA, analysis of variance.
Table S2 Statistical analysis of seroconversion rate and geometric mean titer against poliovirus (types 1, 2, and 3) 28 days after three doses of different immunization schedules in per protocol set analysis

\begin{tabular}{|c|c|c|}
\hline Immunogenicity indicator & Test method & $P$ value \\
\hline \multicolumn{3}{|l|}{ Type 1 polio strain } \\
\hline Seropositive, n (\%, 95\% Cl) & Fisher exact test & 0.72 \\
\hline GMT, geometric mean $(95 \% \mathrm{Cl})$ & ANOVA & $<0.001$ \\
\hline \multicolumn{3}{|l|}{ Type 2 polio strain } \\
\hline Seropositive, n (\%, 95\% Cl) & Pearson CHI-SQUARE & $<0.001$ \\
\hline GMT, geometric mean $(95 \% \mathrm{Cl})$ & ANOVA & $<0.001$ \\
\hline \multicolumn{3}{|l|}{ Type 3 polio strain } \\
\hline Seropositive, n (\%, 95\% Cl) & Fisher exact test & $<0.05$ \\
\hline GMT, geometric mean $(95 \% \mathrm{Cl})$ & ANOVA & $<0.001$ \\
\hline
\end{tabular}


Table S3 Multiple comparisons of seroconversion rate against poliovirus (types 1, 2, and 3) 28 days after three doses of different immunization schedules

\begin{tabular}{|c|c|c|c|c|c|}
\hline Immunogenicity indicator & Group & vs. & Group & Test method & $P$ value \\
\hline \multirow[t]{21}{*}{ Type2 polio strain } & \multirow[t]{6}{*}{ clPV-bOPV-bOPV } & \multirow[t]{6}{*}{ vs. } & sIPV-sIPV-tOPV & Pearson CHI-SQUARE & $<0.001$ \\
\hline & & & sIPV-sIPV-bOPV & Pearson CHI-SQUARE & $<0.001$ \\
\hline & & & cIPV-clPV-tOPV & Pearson CHI-SQUARE & $<0.001$ \\
\hline & & & cIPV-clPV-bOPV & Pearson CHI-SQUARE & $<0.001$ \\
\hline & & & sIPV-bOPV- bOPV & Pearson CHI-SQUARE & 0.13 \\
\hline & & & tOPV-tOPV-tOPV & Pearson CHI-SQUARE & $<0.001$ \\
\hline & \multirow[t]{5}{*}{ sIPV-sIPV-tOPV } & \multirow[t]{5}{*}{ vs. } & sIPV-sIPV-bOPV & Pearson CHI-SQUARE & $<0.001$ \\
\hline & & & clPV-clPV-tOPV & Fisher exact test & 1.00 \\
\hline & & & cIPV-cIPV-bOPV & Pearson CHI-SQUARE & $<0.001$ \\
\hline & & & sIPV-bOPV- bOPV & Pearson CHI-SQUARE & $<0.001$ \\
\hline & & & tOPV-tOPV-tOPV & Fisher exact test & 0.75 \\
\hline & \multirow[t]{4}{*}{ sIPV-sIPV-bOPV } & \multirow[t]{4}{*}{ vs. } & clPV-clPV-tOPV & Pearson CHI-SQUARE & $<0.001$ \\
\hline & & & cIPV-cIPV-bOPV & Pearson CHI-SQUARE & 0.79 \\
\hline & & & sIPV-bOPV- bOPV & Pearson CHI-SQUARE & $<0.001$ \\
\hline & & & tOPV-tOPV-tOPV & Pearson CHI-SQUARE & $<0.001$ \\
\hline & \multirow[t]{3}{*}{ clPV-clPV-tOPV } & \multirow[t]{3}{*}{ vs. } & cIPV-clPV-bOPV & Pearson CHI-SQUARE & $<0.001$ \\
\hline & & & sIPV-bOPV- bOPV & Pearson CHI-SQUARE & $<0.001$ \\
\hline & & & tOPV-tOPV-tOPV & Fisher exact test & 0.75 \\
\hline & \multirow[t]{2}{*}{ clPV-clPV-bOPV } & \multirow[t]{2}{*}{ vs. } & sIPV-bOPV- bOPV & Pearson CHI-SQUARE & $<0.001$ \\
\hline & & & tOPV-tOPV-tOPV & Pearson CHI-SQUARE & $<0.001$ \\
\hline & sIPV-bOPV- bOPV & vs. & tOPV-tOPV-tOPV & Pearson CHI-SQUARE & $<0.001$ \\
\hline \multirow[t]{21}{*}{ Type3 polio strain } & \multirow[t]{6}{*}{ clPV-bOPV-bOPV } & \multirow[t]{6}{*}{ vs. } & sIPV-sIPV-tOPV & Fisher exact test & 0.21 \\
\hline & & & sIPV-sIPV-bOPV & Fisher exact test & 1 \\
\hline & & & clPV-clPV-tOPV & Fisher exact test & 0.62 \\
\hline & & & cIPV-cIPV-bOPV & Fisher exact test & 0.62 \\
\hline & & & sIPV-bOPV- bOPV & Pearson CHI-SQUARE & 1 \\
\hline & & & tOPV-tOPV-tOPV & Pearson CHI-SQUARE & $<0.05$ \\
\hline & \multirow[t]{5}{*}{ sIPV-sIPV-tOPV } & \multirow[t]{5}{*}{ vs. } & sIPV-sIPV-bOPV & Fisher exact test & 0.20 \\
\hline & & & cIPV-clPV-tOPV & Fisher exact test & 0.45 \\
\hline & & & cIPV-cIPV-bOPV & Fisher exact test & 0.69 \\
\hline & & & sIPV-bOPV- bOPV & Fisher exact test & 0.21 \\
\hline & & & tOPV-tOPV-tOPV & Pearson CHI-SQUARE & 0.49 \\
\hline & \multirow[t]{4}{*}{ sIPV-sIPV-bOPV } & \multirow[t]{4}{*}{ vs. } & clPV-clPV-tOPV & Fisher exact test & 0.61 \\
\hline & & & cIPV-cIPV-bOPV & Fisher exact test & 0.61 \\
\hline & & & sIPV-bOPV- bOPV & Fisher exact test & 1 \\
\hline & & & tOPV-tOPV-tOPV & Pearson CHI-SQUARE & $<0.05$ \\
\hline & \multirow[t]{3}{*}{ clPV-clPV-tOPV } & \multirow[t]{3}{*}{ vs. } & cIPV-cIPV-bOPV & Fisher exact test & 1 \\
\hline & & & sIPV-bOPV- bOPV & Fisher exact test & 0.62 \\
\hline & & & tOPV-tOPV-tOPV & Pearson CHI-SQUARE & 0.09 \\
\hline & \multirow[t]{2}{*}{ clPV-cIPV-bOPV } & \multirow[t]{2}{*}{ vs. } & sIPV-bOPV- bOPV & Fisher exact test & 0.62 \\
\hline & & & tOPV-tOPV-tOPV & Pearson CHI-SQUARE & 0.09 \\
\hline & sIPV-bOPV- bOPV & vs. & tOPV-tOPV-tOPV & Pearson CHI-SQUARE & $<0.05$ \\
\hline
\end{tabular}

tOPV, trivalent oral polio vaccine; CIPV, conventional inactivated poliovirus vaccine; bOPV, bivalent oral polio vaccine; sIPV, Sabin strainbased inactivated poliovirus vaccine. 
Table S4 Multiple comparisons of antibody geometric mean titer against poliovirus (types 1, 2, and 3) 28 days after three doses of different immunization schedules

\begin{tabular}{|c|c|c|c|c|c|}
\hline Immunogenicity indicator & Group & vs. & Group & Test method & $P$ value \\
\hline \multirow[t]{21}{*}{ Type1 polio strain } & \multirow[t]{6}{*}{ clPV-bOPV-bOPV } & \multirow[t]{6}{*}{ vs. } & sIPV-sIPV-bOPV & T test & $<0.001$ \\
\hline & & & clPV-cIPV-bOPV & T test & 0.30 \\
\hline & & & sIPV-sIPV-tOPV & T test & $<0.001$ \\
\hline & & & cIPV-cIPV-tOPV & T test & $<0.001$ \\
\hline & & & sIPV-bOPV- bOPV & T test & $<0.001$ \\
\hline & & & tOPV-tOPV-tOPV & T test & $<0.001$ \\
\hline & \multirow[t]{5}{*}{ sIPV-sIPV-bOPV } & \multirow[t]{5}{*}{ vs. } & cIPV-cIPV-bOPV & T test & $<0.001$ \\
\hline & & & sIPV-sIPV-tOPV & T test & $<0.001$ \\
\hline & & & cIPV-cIPV-tOPV & T test & $<0.001$ \\
\hline & & & sIPV-bOPV- bOPV & T test & $<0.01$ \\
\hline & & & tOPV-tOPV-tOPV & T test & $<0.001$ \\
\hline & \multirow[t]{4}{*}{ clPV-clPV-bOPV } & \multirow[t]{4}{*}{ vs. } & sIPV-sIPV-tOPV & T test & $<0.001$ \\
\hline & & & cIPV-cIPV-tOPV & T test & $<0.001$ \\
\hline & & & sIPV-bOPV- bOPV & T test & $<0.001$ \\
\hline & & & tOPV-tOPV-tOPV & T test & $<0.001$ \\
\hline & \multirow[t]{3}{*}{ sIPV-sIPV-tOPV } & \multirow[t]{3}{*}{ vs. } & clPV-cIPV-tOPV & T test & $<0.001$ \\
\hline & & & sIPV-bOPV- bOPV & $\mathrm{T}$ test & 0.08 \\
\hline & & & tOPV-tOPV-tOPV & T test & 0.59 \\
\hline & \multirow{2}{*}{ clPV-clPV-tOPV } & \multirow[t]{2}{*}{ vs. } & sIPV-bOPV- bOPV & T test & $<0.001$ \\
\hline & & & tOPV-tOPV-tOPV & T test & $<0.001$ \\
\hline & sIPV-bOPV- bOPV & vs. & tOPV-tOPV-tOPV & T test & $<0.01$ \\
\hline \multirow[t]{21}{*}{ Type2 polio strain } & \multirow[t]{6}{*}{ clPV-bOPV-bOPV } & \multirow[t]{6}{*}{ vs. } & sIPV-sIPV-bOPV & T test & $<0.001$ \\
\hline & & & cIPV-clPV-bOPV & T test & $<0.001$ \\
\hline & & & sIPV-sIPV-tOPV & T test & $<0.001$ \\
\hline & & & CIPV-cIPV-tOPV & T test & $<0.001$ \\
\hline & & & sIPV-bOPV- bOPV & T test & $<0.001$ \\
\hline & & & tOPV-tOPV-tOPV & T test & $<0.001$ \\
\hline & sIPV-sIPV-bOPV & vs. & cIPV-cIPV-bOPV & T test & 0.53 \\
\hline & & & sIPV-sIPV-tOPV & $\mathrm{T}$ test & $<0.001$ \\
\hline & & & clPV-clPV-tOPV & T test & $<0.001$ \\
\hline & & & sIPV-bOPV- bOPV & T test & $<0.001$ \\
\hline & & & tOPV-tOPV-tOPV & T test & $<0.001$ \\
\hline & cIPV-clPV-bOPV & vs. & sIPV-sIPV-tOPV & T test & $<0.001$ \\
\hline & & & clPV-cIPV-tOPV & T test & $<0.001$ \\
\hline & & & sIPV-bOPV- bOPV & T test & $<0.001$ \\
\hline & & & tOPV-tOPV-tOPV & T test & $<0.001$ \\
\hline & sIPV-sIPV-tOPV & vs. & CIPV-cIPV-tOPV & T test & 0.17 \\
\hline & & & sIPV-bOPV- bOPV & T test & $<0.001$ \\
\hline & & & tOPV-tOPV-tOPV & T test & $<0.001$ \\
\hline & CIPV-cIPV-tOPV & vs. & sIPV-bOPV- bOPV & T test & $<0.001$ \\
\hline & & & tOPV-tOPV-tOPV & T test & $<0.001$ \\
\hline & sIPV-bOPV- bOPV & vs. & tOPV-tOPV-tOPV & T test & $<0.001$ \\
\hline Type3 polio strain & cIPV-bOPV-bOPV & vs. & sIPV-sIPV-bOPV & T test & $<0.001$ \\
\hline & & & cIPV-cIPV-bOPV & T test & $<0.001$ \\
\hline & & & sIPV-sIPV-tOPV & T test & $<0.05$ \\
\hline & & & cIPV-cIPV-tOPV & T test & $<0.05$ \\
\hline & & & sIPV-bOPV- bOPV & T test & 0.95 \\
\hline & & & tOPV-tOPV-tOPV & T test & $<0.001$ \\
\hline & sIPV-sIPV-bOPV & vs. & cIPV-cIPV-bOPV & T test & 0.68 \\
\hline & & & sIPV-sIPV-tOPV & T test & $<0.001$ \\
\hline & & & clPV-cIPV-tOPV & T test & $<0.001$ \\
\hline & & & sIPV-bOPV- bOPV & T test & $<0.001$ \\
\hline & & & tOPV-tOPV-tOPV & T test & $<0.001$ \\
\hline & cIPV-cIPV-bOPV & vs. & sIPV-sIPV-tOPV & T test & $<0.01$ \\
\hline & & & cIPV-cIPV-tOPV & T test & $<0.001$ \\
\hline & & & sIPV-bOPV- bOPV & T test & $<0.001$ \\
\hline & & & tOPV-tOPV-tOPV & T test & $<0.001$ \\
\hline & sIPV-sIPV-tOPV & vs. & cIPV-cIPV-tOPV & T test & 0.82 \\
\hline & & & sIPV-bOPV- bOPV & T test & $<0.05$ \\
\hline & & & tOPV-tOPV-tOPV & T test & $<0.001$ \\
\hline & clPV-clPV-tOPV & vs. & sIPV-bOPV- bOPV & T test & $<0.05$ \\
\hline & & & tOPV-tOPV-tOPV & T test & $<0.001$ \\
\hline & sIPV-bOPV- bOPV & vs. & tOPV-tOPV-tOPV & $\mathrm{T}$ test & $<0.001$ \\
\hline
\end{tabular}

tOPV, trivalent oral polio vaccine; cIPV, conventional inactivated poliovirus vaccine; bOPV, bivalent oral polio vaccine; sIPV, Sabin strainbased inactivated poliovirus vaccine. 
Table S5 Statistical analysis of incidence of adverse events in the infants after immunization with different polio vaccination schedules in safety set analysis

\begin{tabular}{|c|c|c|}
\hline Adverse event & Test method & $P$ value \\
\hline Any adverse event & Pearson CHI-SQUARE & 0.60 \\
\hline Serious adverse events & Pearson CHI-SQUARE & 0.97 \\
\hline Unsolicited adverse events & Pearson CHI-SQUARE & $<0.001$ \\
\hline Solicited adverse events & Pearson CHI-SQUARE & $<0.001$ \\
\hline Fever & Pearson CHI-SQUARE & $<0.001$ \\
\hline Abnormal irritability & Pearson CHI-SQUARE & $<0.001$ \\
\hline Drowsiness & Pearson CHI-SQUARE & $<0.001$ \\
\hline Gastrointestinal symptoms & Pearson CHI-SQUARE & $<0.05$ \\
\hline Diarrhea & Pearson CHI-SQUARE & 0.59 \\
\hline Allergy & Fisher exact test & 0.09 \\
\hline Local reactions $^{c}$ & Pearson CHI-SQUARE & $<0.001$ \\
\hline
\end{tabular}

${ }^{\mathrm{c}}$, including pain, redness, swelling, and induration. 